\title{
Idiopathic Arachnoiditis of the Cauda Equina: A Case Report from Tanzania
}

\author{
Emmanuel V. Assey ${ }^{a} \quad$ Abid M. Sadiq ${ }^{a, c}$ Magreth J. Swai ${ }^{c}$ \\ Adnan M. Sadiq ${ }^{\text {b, c }}$ Marieke C.J. Dekker ${ }^{a, c}$ \\ aDepartment of Internal Medicine, Kilimanjaro Christian Medical Centre, Moshi, Tanzania; \\ ${ }^{\text {b}}$ Department of Radiology, Kilimanjaro Christian Medical Centre, Moshi, Tanzania; \\ 'Kilimanjaro Christian Medical University College, Moshi, Tanzania
}

\section{Keywords}

Arachnoiditis · Cauda equina - Magnetic resonance imaging $\cdot$ Young adult

\begin{abstract}
Arachnoiditis is a rare clinical entity that usually presents with severe debilitating radiating pain that can pose a challenge in diagnosis especially in areas without appropriate imaging modalities. We present a 26 -year-old male with progressive lower back pain with radiation to the lower extremities, aggravated by movement and touch. We diagnosed idiopathic arachnoiditis based on cerebrospinal fluid analysis and magnetic resonance imaging. He was managed with a 5-day course of methylprednisolone and analgesics with a good outcome. Severe back pain without a specific cause in a young patient should be investigated with proper imaging modalities and lumbar puncture if warranted to evaluate the cause.
\end{abstract}

(c) 2021 The Author(s).

Published by S. Karger AG, Basel

\section{Introduction}

Arachnoiditis is a rare disorder causing chronic pain because of scar tissue build-up, from the inflammatory process, causing adhesion of the nerve roots to the arachnoid lining of the meninges [1]. The pathophysiology includes invasion of fibrinous and oligo-cellular exudates that result from inflammatory processes in the pia arachnoid layer as a result of an initial insult [2]. Such insults could be a result of tuberculosis (TB), syphilis, spinal trauma, neoplasms, surgery, lumbar puncture, or intrathecal injections of the spine. There have been other reports of subarachnoid hemorrhage, myelographic contrast agents, degeneration of the spinal cord, genetic fibrinolytic defects, and keloid-like scarring $[3,4]$. In some rare instances, no specific cause can be demonstrated leading to conceivable diag- 
noses such as mechanical irritation, undiagnosed TB, or an aseptic inflammation [5]. TB arachnoiditis should be considered in endemic regions especially when no other obvious cause is found [6].

The presentation of the disease can range from mild to debilitating pain and even result in long-term disability with paraplegia [7]. The case presented here is of a man with severe pain diagnosed with adhesive arachnoiditis using magnetic resonance imaging (MRI) who benefited from a short course of corticosteroid treatment.

\section{Case Report}

We present a case of a 26-year-old, otherwise healthy man who reported gradual progressive back pain for a period of 3 months. It radiated to the lower thighs and occasionally to the feet, with no report of lower limb weakness. The pain was worse upon movement of his legs, hips, and lower back. He had no history of chronic back pain or bladder or bowel dysfunction. He was working as a soldier in training. He denied any history of spinal injections or procedures. He did not use alcohol, tobacco, or any illicit drugs.

Upon examination, he was clinically pale and in severe pain with visual analog scale score 10/10. His vital signs were normal, and there was no fever. There were no skin lesions or lymphadenopathy. Higher cognitive function, cranial nerves, muscle bulk, tone, power, and reflexes were normal. No fasciculations were seen, and the sphincter tone was normal. The sensory examination of the upper and lower limbs was normal in temperature and proprioception; however, he had severe allodynia on the lower back and lower limbs generally. He experienced severe pain on light touch of the lower truncal region, and however, the pain did not follow any dermatome pattern.

A full blood count showed hemoglobin of $8.1 \mathrm{~g} / \mathrm{dL}$, mild leukocytosis, and an elevated erythrocyte sedimentation rate of $105 \mathrm{~mm} / \mathrm{h}$. Renal and liver function tests together with electrolytes were all found to be within the normal range.

Lumbar puncture results showed a clear cerebrospinal fluid (CSF) with an elevated protein of $945.0 \mathrm{mg} / \mathrm{L}$ and glucose of $31 \mathrm{mg} / \mathrm{dL}$ with no pleocytosis. GeneXpert polymerase chain reaction for Mycobacterium tuberculosis on CSF was negative. Cytology on the CSF revealed no abnormal cells. Both serum and CSF rapid plasma reagin for syphilis were negative. His serology for human immunodeficiency virus was negative. A chest $\mathrm{X}$-ray was normal. MRI of the lumbar spine shows thickened, clumped, and displaced cauda equina nerve roots suggestive of a chronic inflammatory process (Fig. 1, 2).

Because of the severity of the pain, analgesic treatment was escalated to morphine, and there was only mild relief from added pregabalin and amitriptyline. Because his CSF protein levels were higher than normal, he was initiated on intravenous methylprednisolone $1 \mathrm{~g}$ daily for 5 days then proceeded to oral prednisolone $60 \mathrm{mg}$ for another 5 days with gradual tapering of the dose over 6 weeks. There was insufficient evidence to start him on anti-TB treatment. On the third day of the methylprednisolone, he showed significant clinical improvement, with reduced pain to visual analog scale $6 / 10$, and could mobilize normally again. He was later discharged home with daily physical therapy and neurology clinic follow-up appointment. On follow-up in the clinic over 3 months, the pain had subsided and he reported no more complaints. Follow-up after 6 months, he reported no neurological complaints and was back to his normal work schedule training as a soldier.

\section{Karger'}



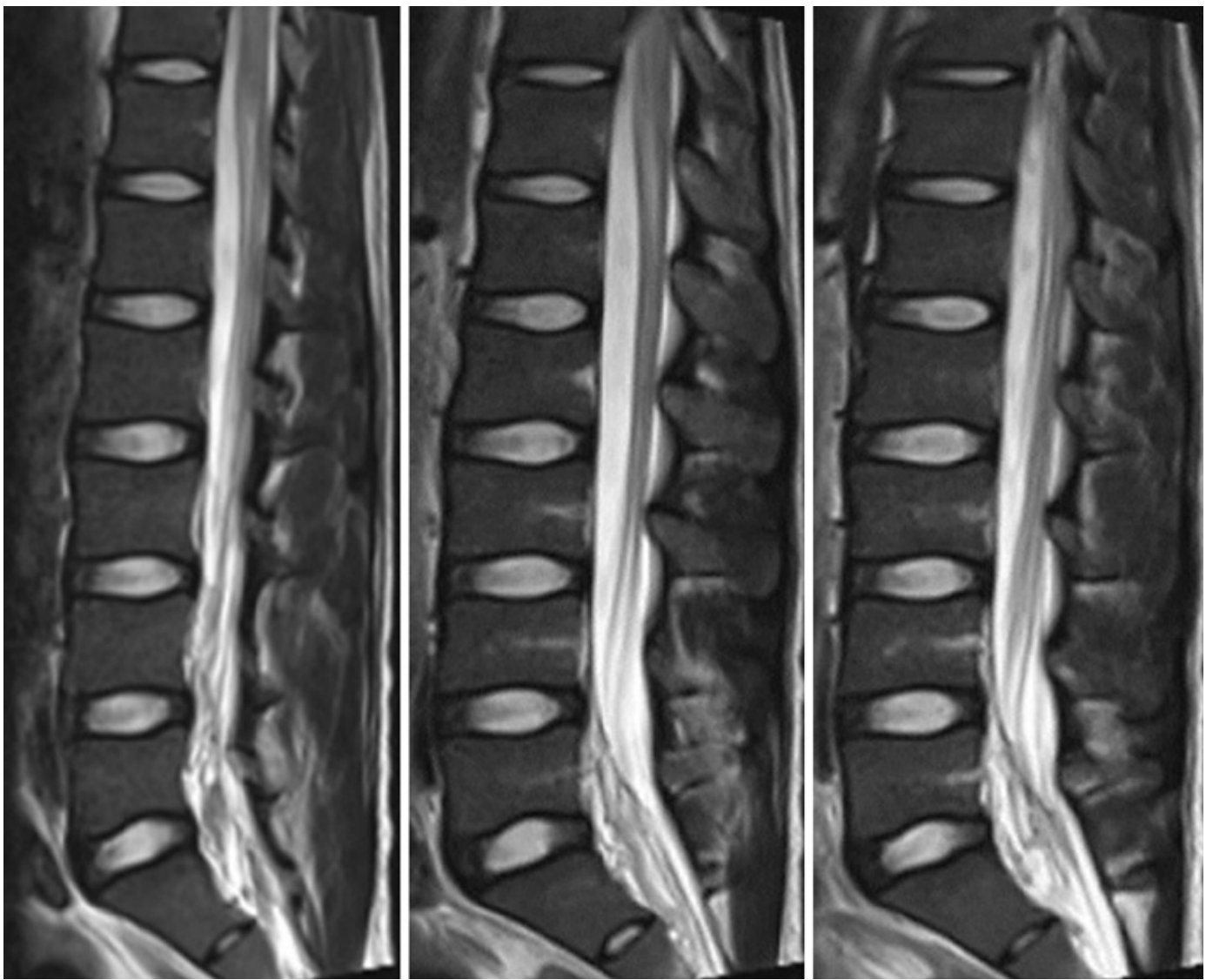

Fig. 1. Sagittal T2-weighted images showing thickened, clumped, and displaced cauda equina nerve roots with altered T2 signal intensity at L5/S1.

\section{Discussion}

In our case, we describe a young male presenting with a 3-month history of severe lower back pain, elevated CSF protein levels with cauda equina thickening seen on MRI. Infectious causes were ruled out with the available means. He was diagnosed with adhesive arachnoiditis and had a fairly good response to pulse treatment of high-dose intravenous corticosteroid therapy.

The common clinical symptoms of arachnoiditis are intractable lower back pain with or without leg pain and paresthesia. Other symptoms include leg weakness, sphincter and sexual dysfunction, and sudden leg weakness mimicking collapse [8]. These symptoms can be those of nerve root compression of the cauda equina hence can be identified as other spinal pathologies such as cauda equina syndrome and inflammatory neuropathies. The severity of the pain can be mistaken for a functional disorder because of the nondermatome distribution pattern and intensity [9]. In a clinical review of 63 patients, $83 \%$ had lumbar-sacral arachnoiditis, while the rest had either thoracic or cervical forms [10]. In our case, anemia of chronic disease is caused by suppression of erythropoiesis from anti-inflammatory cytokine and hormone production [11].

Typical chronic inflammatory demyelinating polyneuropathy (CIDP), of $>8$ weeks from the time of onset, is a symmetrical sensorimotor polyneuropathy with proximal and distal involvement with gradually progressive symptoms over several months. It has been 

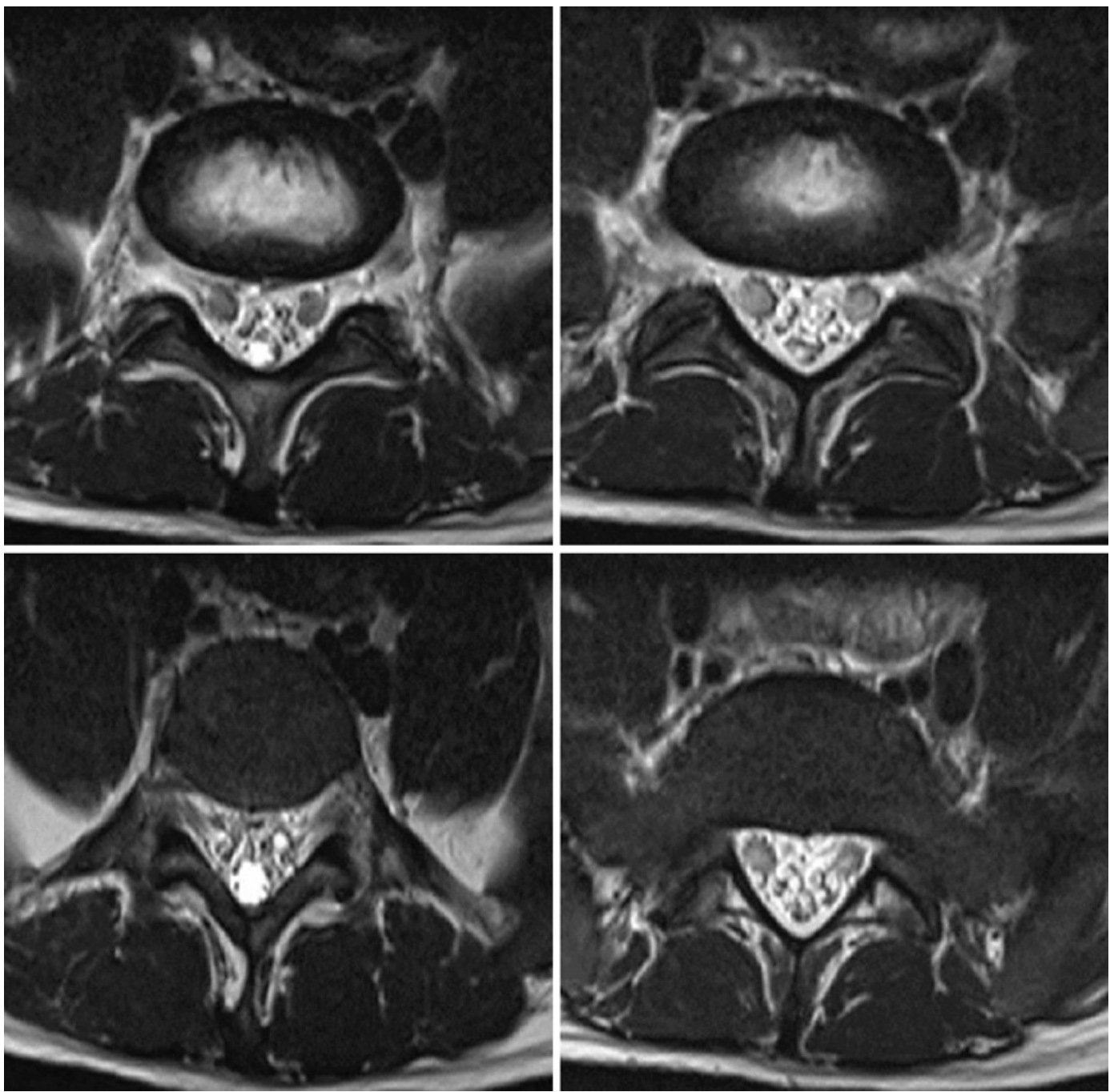

Fig. 2. Axial T2-weighted images showing thickened, clumped, and displaced cauda equina nerve roots.

reported that CIDP may present with an acute onset rather than gradual progression over several months [12]. Variants of CIDP have been documented of atypical nature being either motor, sensory, or focal [12], but in this case, the likely differential diagnosis would be chronic immune sensory polyradiculopathy. The diagnostic criteria for chronic immune sensory polyradiculopathy include sensory symptoms with polyneuropathy without weakness with normal sensory and motor nerve conduction study results, and at least 2 of the following; abnormal somatosensory evoked potentials, MRI showing thickened nerve roots, and/or elevated CSF protein levels with normal cells [13]. The other differential diagnoses for spinal arachnoiditis include spinal cord tumor, syringomyelia, and failed back surgery syndrome [4]. In advanced arachnoiditis, the disease tends to mimic other spinal cord diseases.

The characteristics for arachnoiditis on MRI include clumping and enhancement of nerve roots, arachnoid cysts with cord mass effect and swelling, increased T2 signal, dural or pial enhancement, cord atrophy, arachnoid cord displacement, and syrinx formation [14]. In our case, we saw thickening, clumping, and displacement of the nerve roots with increased T2 signal intensity (Fig. 1, 2). Nerve conduction studies and electromyography 
can differentiate the disease from other forms of polyneuropathy, especially when there is no motor involvement [5]. Unfortunately, nerve conduction studies were not available at the time of this study.

Treatment guidelines for arachnoiditis include the use of steroids, pain relief, and physical therapy. Other interventions include adhesiolysis and radiofrequency rhizotomy although in extreme cases [15]. Our patient had health insurance allowing for quick diagnosis and rapidly recovered, which might be unusual in our setting where a very small minority population are insured against health expenses. And an MRI costs over 3 monthly wages for the average Tanzanian [16].

In conclusion, lumbosacral arachnoiditis is a rare clinical entity and warrants a higher index of suspicion because of its myriad causes, some of which are treatable. The response to therapy is varied and some patients may end up with a disabling weakness or longstanding pain. More studies are required to better understand the best treatment modalities, especially in resource-limited areas.

\section{Acknowledgements}

We thank the patient for his patience and cooperation.

\section{Statement of Ethics}

The authors' institutions do not require ethical approval for the publication of a single case. We obtained written informed consent from the patient for the publication of this case report and the accompanying images.

\section{Conflict of Interest Statement}

The authors have no conflicts of interest to declare.

\section{Funding Sources}

The authors did not receive any funding.

\section{Author Contributions}

E.S., A.S., M.S., and M.D. were involved with patient management; E.S., A.S., and M.S. reviewed the inherent literature; E.S. and A.S. prepared the manuscript; A.S. provided the images; M.D. reviewed and edited the manuscript; all the authors approved the final version of the manuscript.

\section{Data Availability Statement}

No data set was generated for this study.

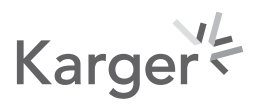




\section{References}

1 Patel R, Agarwal D. Arachnoiditis. Pain. Cham: Springer International Publishing; 2019. p. 647-9.

2 Killeen T, Kamat A, Walsh D, Parker A, Aliashkevich A. Severe adhesive arachnoiditis resulting in progressive paraplegia following obstetric spinal anaesthesia: a case report and review. Anaesthesia. 2012 Dec;67(12): 1386-94.

3 Duke RJ, Hashimoto SA. Familial spinal arachnoiditis. A new entity. Arch Neurol. 1974 Apr 1;30(4):300.

4 Wright MH, Denney LC. A comprehensive review of spinal arachnoiditis. Orthop Nurs. 2003 May;22(3):215-9.

5 Di Ieva A, Barolat G, Tschabitscher M, Rognone E, Aimar E, Gaetani P, et al. Lumbar arachnoiditis and thecaloscopy: brief review and proposed treatment algorithm. Cent Eur Neurosurg. 2010 Nov 21;71(4): 71207-12.

6 Konar SK, Narasinga Rao K, Mahadevan A, Devi BI. Tuberculous lumbar arachnoiditis mimicking conus cauda tumor: a case report and review of literature. J Neurosci Rural Pract. 2011 Jan 26;02(01):93-6.

7 Jurga S, Szymańska-Adamcewicz 0, Wierzchołowski W, Pilchowska-Ujma E, Urbaniak Ł. Spinal adhesive arachnoiditis: three case reports and review of literature. Acta Neurol Belg. 2021 Feb 24;121(1):47-53.

8 Goodman BP. Disorders of the cauda equina. Continuum. 2018 Apr;24(2):584-602.

9 Wright MG. Lumbo-sacral adhesive arachnoiditis. J R Soc Med. 1990 Oct 27;83(10):673.

10 Mooij JJA. Spinal arachnoiditis: disease or coincidence? Acta Neurochir. 1980 Sep;53(3-4):151-60.

11 Madu AJ, Ughasoro MD. Anaemia of chronic disease: an in-depth review. Med Princ Pract. 2017;26(1):1-9.

12 Ruts L, van Koningsveld R, van Doorn PA. Distinguishing acute-onset CIDP from Guillain-Barré syndrome with treatment related fluctuations. Neurology. 2005 Jul 12;65(1):138-40.

13 Doneddu PE, Cocito D, Manganelli F, Fazio R, Briani C, Filosto M, et al. Atypical CIDP: diagnostic criteria, progression and treatment response. Data from the Italian CIDP database. J Neurol Neurosurg Psychiatry. 2019 Feb; 90(2):125-32.

14 Anderson TL, Morris JM, Wald JT, Kotsenas AL. Imaging appearance of advanced chronic adhesive arachnoiditis: a retrospective review. AJR Am J Roentgenol. 2017 Sep;209(3):648-55.

15 Nguyen D, Przkora R. An evidence-based assessment and treatment plan for arachnoiditis. J Pain. 2013 Apr; 14(4):S31.

16 Sadiq AM, Mariki DE, Gundah CM, Assey EV, Zwetselaar M, Howlett WP, et al. Feeling the price tag of magnetic resonance imaging claustrophobia. J Magn Reson Imaging.2021;54(1):328. 